\title{
Los principios éticos y los enfoques asociados a la investigación de la gobernanza en los sistemas de salud: implicaciones conceptuales y metodológicas

\author{
Ethical principles and approaches to health systems governance \\ research: conceptual and methodological implications
}

\author{
Walter Flores
}

Centro de Estudios para la Equidad y Gobernanza en los Sistemas de Salud. Guatemala. wflores@cegss.org.gt

Recibido 15 Julio 2009/Enviado para Modificación 20 Diciembre 2009/Aceptado 15 Enero 2010

\section{RESUMEN}

La gobernanza se refiere a procesos de toma de decisión en los cuales confluyen relaciones de poder e intereses particulares de actores e instituciones. Situaciones de consenso y conflicto son inherentes a dichos procesos. En adición, la toma de decisión sucede dentro de un marco de principios éticos, motivaciones e incentivos que pueden ser explícitos o implícitos. Los sistemas de salud, en la mayoría de países de América Latina y el Caribe, definen los principios de equidad, solidaridad, participación social y derecho a la salud como rectores. Por lo tanto, estos principios deben presidir los procesos de gobernanza. Sin embargo, este no es siempre el caso y es allí donde reside la importancia de investigar la gobernanza en los sistemas de salud. Avanzar en la investigación de la gobernanza tiene implicaciones conceptuales y metodológicas. A nivel conceptual, es de relevancia clarificar e integrar los enfoques normativos y analíticos puesto que ambos son necesarios para un abordaje que busque indagar y comprender la complejidad de los fenómenos sociales. En lo metodológico, es necesario expandir la gama de variables, fuentes de información e indicadores para estudiar la toma de decisiones relacionadas a lograr una mayor equidad, ejercicios de ciudadanía en salud y eficiencia en las políticas públicas.

Palabras Clave: Gobernanza, gobierno, sistemas de salud, poder, América Latina, equidad (fuente: DeCS, BIREME).

\section{ABSTRACT}

Governance refers to decision-making processes in which power relationships and actors and institutions' particular interests converge. Situations of consensus and conflict are inherent to such processes. Furthermore, decision-making happens within a framework of ethical principles, motivations and incentives which could be explicit or implicit. Health systems in most Latin-American and Caribbean countries take the principles of equity, solidarity, social participation and the right to health as 
their guiding principles; such principles must thus rule governance processes. However, this is not always the case and this is where the importance of investigating governance in health systems lies. Making advances in investigating governance involves conceptual and methodological implications. Clarifying and integrating normative and analytical approaches is relevant at conceptual level as both are necessary for an approach seeking to investigate and understand social phenomena's complexity. In relation to methodological level, there is a need to expand the range of variables, sources of information and indicators for studying decision-making aimed to greater equity, health citizenship and public policy efficiency.

Key Words: Governance, government, health systems, power, Latin America (source: MeSH, NLM).

$\mathrm{E}$

l concepto "gobernanza", incluyendo las acepciones "buena gobernanza" y "gobernanza democrática", están de moda de la misma forma que "capital social" y "empoderamiento" lo estuvieron hace algunos años.

Aun cuando el concepto de gobernanza puede quedarse solamente como otro término pasajero en la literatura del desarrollo, también existe la oportunidad de hacerlo útil para describir y analizar interacciones complejas que afectan el desarrollo social y económico. Este artículo parte de la siguiente premisa: El concepto de gobernanza, particularmente aplicado a sistemas de salud, nos permite indagar aspectos y actores sociales que tradicionalmente han estado fuera de los análisis convencionales. Sin embargo, para hacer esto necesitamos tener claridad sobre las diferentes acepciones del concepto, los principios éticos que están en juego y las implicaciones para realizar ejercicios investigativos.

Esta idea será desarrollada a partir de los siguientes cuatro objetivos: a. Abordar la forma en que se ha conceptualizado la gobernanza en los sistemas de salud; b. Identificar los principios éticos que sustentan el análisis de la gobernanza en los sistemas de salud; c. Discutir los enfoques normativos y analíticos en la investigación sobre gobernanza como dos vertientes necesarias y complementarias; y d. Discutir la forma en que los principios y valores éticos permean el cuerpo de indicadores, variables y herramientas que se aplican en la investigación sobre gobernanza en sistemas de salud. Antes de ello, se aclara que en la literatura en idioma Español se usan indistintamente los conceptos gobernanza, gobernabilidad y gobernancia. En este artículo se ha preferido usar el concepto gobernanza, el cual tiene una mayor congruencia con el de "governance" que aparece en la literatura en idioma Inglés.

Previo a abordar la gobernanza en los sistemas de salud, es necesario hacer referencia a las corrientes relacionadas con la gobernanza en los sistemas sociopolíticos y hacer explícita la corriente en la cual el autor se 
apoya. La literatura en el tema es basta y existe una gran cantidad de clasificaciones y corrientes. Sin embargo, para los propósitos del presente artículo, se decidió presentar tres corrientes que parecen diferenciarse claramente en la literatura. La primera es el uso que hacen los organismos internacionales de cooperación financiera, quienes se preocupan por la "buena gobernanza". En la gran mayoría de casos, la buena gobernanza significa poner en práctica reformas institucionales (por ejemplo la liberalización de las economías y la reducción del papel del Estado) que estas agencias han recomendado a los países en las últimas dos décadas (1). La hegemonía que ha tenido esta visión de buena gobernanza cae en el etnocentrismo cuando sostiene implícitamente, que la buena gobernanza se refiere a seguir prácticas de las economías y sociedades occidentales (2).

La segunda corriente aborda la gobernanza dentro de los procesos de descentralización política y administrativa que se han realizado en varios países. Esta corriente se caracteriza por hacer énfasis en la gobernanza como el conjunto de herramientas administrativas para la gestión pública. En otras palabras, se presentan como procesos técnicos y apolíticos (3). La tercera sostiene que aún cuando la gobernanza incluye herramientas técnicas, ésta es en esencia un proceso político. La gobernanza como proceso debe servir para implementar reformas y políticas públicas que promuevan mayores niveles de equidad, igualdad ante la ley y justicia social (4-6). Varios autores definen esta corriente como "gobernanza democrática" para diferenciarla de las otras corrientes $(6,7)$.

El presente trabajo se afilia a la corriente de la gobernanza democrática, la cual se fundamenta en una serie de principios éticos y objetivos sociales que buscan ser alcanzados a través de las políticas públicas. Dichos principios y objetivos son discutidos y aplicados a la investigación sobre gobernanza en los sistemas de salud en las secciones posteriores.

\section{El concepto de gobernanza aplicado a los sistemas de salud}

La aplicación del concepto de Gobernanza a los sistemas de salud tiene ya más de una década. En 1998, la Organización Mundial de la Salud (OMS) la definió como: "La participación de actores a los cuales les concierne la definición e implementación de políticas, programas y prácticas que promueven sistemas de salud equitativos y sostenibles" (8). 
La definición de la OMS es limitada porque asocia a la gobernanza únicamente con participación de actores. A través del desarrollo conceptual de gobernanza en las ciencias políticas y en el desarrollo económico social, se sabe que aun cuando los actores son parte de los procesos de gobernanza, las reglas del juego (formales y no formales) y las organizaciones son también partes esenciales del proceso $(5,7)$.

Es posible decir que en esencia, la gobernanza se refiere a procesos de toma de decisión. Por lo tanto, las relaciones de poder juegan un papel central en su estudio. Por esto, el Programa de Naciones Unidas para el Desarrollo situó "el poder" como un eje central de su definición de gobernanza: "...La gobernanza incluye todos los métodos -sean buenos o malos- que las sociedades utilizan para distribuir el poder y gestionar los recursos públicos y problemas..." (6).

La definición del PNUD no es específica a los sistemas de salud sino al desarrollo en general. Sin embargo, si se toma en cuenta ambas definiciones (OMS y PNUD) es posible inferir que en el análisis de la gobernanza se debe identificar los espacios de toma de decisión, a los actores y a las organizaciones que participan, cuáles son las reglas del juego y la forma en que se expresan las relaciones de poder. Por lo tanto, en este trabajo se propone una definición que sintetiza los temas anteriores. La gobernanza en los sistemas de salud se refiere a: Los procesos para la toma de decisiones que afectan de forma directa o indirecta al sistema de salud. En dichos procesos interactúan actores estratégicos y organizaciones a través de reglas de juego y otros procedimientos (formales e informales). Los actores estratégicos utilizan recursos de poder para influir en la de toma de decisiones.

Es importante notar que en la definición anterior que se ha propuesto, el "poder" es central para explicar los procesos que influyen en los aspectos y temas que finalmente son decididos y que afectarán al sistema de salud. Las decisiones pueden no satisfacer a todos los actores, así como puede que tampoco sean en beneficio de la mayoría, o que no estén en línea con algún objetivo social de política pública como promover la equidad, solidaridad y eficiencia en el sistema de salud. En otras palabras, aun cuando es deseable que las decisiones siempre sean satisfactorias y de beneficio para todos los interesados, este no es siempre el caso, habrá ganadores y perdedores. Algunas decisiones específicas (como la compra de tecnología e insumos médicos, o la reasignación de presupuestos públicos a territorios geográficos) generarán mayor oposición o conflictividad que otras. Entender la forma en que el "poder" y las relaciones de poder afectan los procesos de toma de decisión es esencial. 
Si el "poder" y las relaciones de poder son centrales a los procesos de gobernanza, entonces también es importante entender cómo se ha conceptualizado el poder y la forma en que éste se expresa en las relaciones sociales. La literatura al respecto es extensa y no es el propósito de este artículo repasarla. Por lo tanto se hará referencia únicamente a dos temas que son relevantes para entender las relaciones de poder en los procesos de toma de decisión.

El primer tema se refiere al poder y su capacidad para producir cambios en las sociedades. Dichos cambios pueden ser resultados del conflicto o del consenso entre los actores sociales que detentan las cuotas de poder (913).El segundo tema se refiere a entender el poder de una forma dinámica donde éste es una capacidad en latencia y la "influencia" es el ejercicio de ese poder. Para convertir esa capacidad latente en influencia en el proceso de toma de decisiones, se requiere accionarlo y en algunas ocasiones, el cobrar conciencia sobre dicha capacidad. Debido a que existe un poder en potencia o latente en todos los individuos, el poder no es suma-cero. Cualquier grupo poblacional puede, en determinado momento, decidir ejercer el poder 'latente' y convertirlo en un poder efectivo (14).

La visión del poder en 'latencia' sirve para explicar la situación social reciente de algunos países de la región (Argentina con el movimiento de piqueteros, Bolivia con el movimiento de campesinos indígenas y sindicatos y Ecuador con el movimiento indígena) en donde los grupos socialmente excluidos y desposeídos cobran 'conciencia' del poder 'latente' y lo hacen efectivo para lograr un reacomodo del poder y generar cambios en las políticas sociales y en algunos casos en la misma estructura social (15-17).

Las relaciones de poder no son mecánicas y en determinadas circunstancias, la correlación de fuerzas puede cambiar rápidamente e incluir como actor estratégico (con recursos de poder) a aquellos que usualmente no lo son (por ejemplo grupos poblacionales socialmente excluidos). Si lo aplicamos a las decisiones que afectan a los sistemas de salud, el análisis de las relaciones de poder es vital para indagar por qué algunos países de la región han adoptado diferentes esquemas de reformas a sus sistemas de salud (por ejemplo, la privatización y el subcontrato de servicios a entes privados versus la expansión y fortalecimiento de la red pública de servicios). Igualmente relevante es indagar de qué forma llegaron representantes de la ciudadanía a ser actores estratégicos en los sistemas de salud (como es el caso de los consejos municipales de salud en Brasil) y cuáles son los beneficios 
de la participación de esos actores en comparación con otros sistemas cuyas decisiones siguen siendo dominadas por actores tradicionales (autoridades centrales de los ministerios de salud, asociaciones gremiales y la industria médica entre otros).

\section{Principios éticos asociados al análisis de la gobernanza en los sistemas de salud}

En general, los sistemas de salud de los países de América Latina y el Caribe están fundamentados en los principios de equidad, igualdad (de oportunidades y responsabilidades ante la ley) solidaridad y la participación social. La mayoría de países también reconocen el derecho a la salud a nivel de sus constituciones políticas. Por lo tanto, siendo la gobernanza procesos de toma de decisiones que afectan en forma directa e indirecta al sistema de salud, es obvio que dichos procesos de toma de decisión deben ser regidos por los mismos principios de equidad, igualdad y solidaridad. Por ello, un tema central en la investigación de la gobernanza en los sistemas de salud es indagar de qué forma los procesos de toma de decisión están alineados a estos principios éticos. Además, es importante tomar en cuenta que dichos principios se convierten al mismo tiempo en objetivos sociales para los sistemas de salud: Mayor equidad en el acceso y financiamiento del sistema de salud, igualdad (ante la ley) de oportunidades y responsabilidades para contribuir financieramente al sistema y la participación social en las políticas públicas de salud, incluyendo la asignación de recursos públicos.

Es importante tener claro que debido a las relaciones de poder, el que exista un marco ético en las políticas públicas no asegura de forma automática su aplicación en los procesos de toma de decisión. Por lo contrario, es frecuente encontrar que las decisiones adoptadas contradicen las metas sociales y ocasionan barreras para la equidad, la solidaridad y una desmejora en la eficiencia en el uso y asignación de recursos (entre otras). Las reglas del juego, los intereses particulares de los actores y las organizaciones (sean públicos o privados) juegan un papel determinante. Por ello, la indagación debe sobrepasar el análisis meramente formalista o superficial y buscar una profundización que permita la comprensión de los valores, motivaciones, incentivos y las prácticas de los actores sociales que participan en los procesos de toma de decisión. Esto incluye la expansión del análisis hacia aquellos grupos sociales que debido a condiciones de exclusión social (sea por condiciones de pobreza, etnicidad, género u otros), no son usualmente tomados en cuenta por no ser considerados actores estratégicos. 


\section{Enfoques normativos y analíticos en el análisis de la Gobernanza}

En las secciones anteriores se ha discutido el concepto de gobernanza en los sistemas de salud y los principios éticos que lo sostiene. Ello forma la base para el desarrollo del instrumental metodológico (variables, indicadores, técnicas de recolección de datos y análisis) que es aplicado en el estudio de la gobernanza en los sistemas de salud. En la literatura sobre investigación en gobernanza, aparecen dos enfoques que se presentan como diferentes para indagar el tema de gobernanza: El enfoque normativo y el enfoque analítico. Dichos enfoques se describen brevemente a continuación.

Un enfoque normativo en el análisis de gobernanza se caracteriza por emitir juicios de valor acerca de las prácticas de los actores sociales y las organizaciones. Ejemplos de análisis normativos son las evaluaciones de "buena gobernanza" que realizan algunas agencias internacionales de cooperación. Varios autores han sido críticos de esos estudios y han indicado que los enfoques normativos estigmatizan a las sociedades, particularmente aquellas en desarrollo, y no reflejan los procesos que suceden alrededor de la interacción de actores, las reglas y la toma de decisiones (2).

Un enfoque analítico de la gobernanza hace énfasis en la aplicación de instrumentos analíticos y metodológicos para entender los procesos de cambio, sin especificar si los procesos de cambio investigados son deseables o justos para alcanzar los objetivos sociales que se proponen las organizaciones y los actores. Ejemplos de enfoques analíticos son la propuestas de Court (18) y la de Hufty (19).

Estos dos enfoques, que a veces son presentados como mutuamente excluyentes, son en realidad dos vertientes necesarias y complementarias en los análisis de gobernanza. El enfoque analítico permite estudiar cuáles son las prácticas, formas y estrategias de interacción de los actores estratégicos, los arreglos organizacionales y reglas del juego en relación a las variables e indicadores predefinidos (es decir, no en el vacío sino bajo un marco de principios y valores implícitos). El enfoque normativo permite identificar cuáles son la prácticas sociales deseables para alcanzar los objetivos sociales que buscamos y también cuales son las prácticas que minan la posibilidad de alcanzar dichos objetivos $(5,7)$.

Lo que se desea resaltar con lo anterior es el hecho que aunque se proponga que un ejercicio investigativo es analítico y no normativo, en realidad 
lo que sucede es que tiene un énfasis en el aspecto analítico pero aún no se desliga de lo normativo. Esto ocurre porque aunque no se emitan juicios de valor, los indicadores y variables predefinidas para investigar el tema de gobernanza tiene implícitos una serie de valores y principios éticos. Por otro lado, un ejercicio investigativo desde el punto de vista normativo necesita aplicar variables e indicadores para identificar y valorar las prácticas de gobernanza implementadas por los actores sociales e instituciones.

En resumen, más que discutir si un ejercicio investigativo es puramente normativo o analítico, es de mayor importancia que los investigadores hagan explícitos los principios y valores que sostienen el marco conceptual que se aplica y que exista correspondencia entre dicho marco conceptual y el cuerpo analítico y metodológico que se aplica para investigar el fenómeno. Esto es muy importante porque existen investigaciones en el tema de gobernanza que se presentan como enfoques analíticos exclusivos, cuando en realidad pueden claramente identificarse una variedad de valores y principios implícitos que sustentan dichos análisis que van desde principios y valores 'neoliberales' hasta aquellos sustentados en desarrollo humano, equidad, igualdad y justicia social $(1,4,5,20)$. En otras palabras, debemos superar el aparente debate entre lo normativo versus lo analítico para concentrarnos en el desarrollo de marcos conceptuales y el instrumental metodológico que nos permita realizar ejercicios investigativos con rigor.

\section{Selección, desarrollo y aplicación de indicadores y variables para el estudio gobernanza}

La selección y aplicación de un cuerpo metodológico (variables, indicadores, técnicas de recolección y análisis de datos) en la investigación de gobernanza está influenciado (de forma explicita o implícita) en principios y valores y los objetivos sociales que se buscan alcanzar a través de las políticas públicas. Por ejemplo, si se fuera a aplicar la variable: "marco legal y certeza jurídica", se puede aplicar indicadores específicos como "existencia de leyes que sustentan respeto a la propiedad privada" y también "existencia de mecanismos legales para proteger a la población desempleada de los gastos catastróficos en salud". El primer indicador es uno de los indicadores estándar de los enfoques neoliberales mientras que el segundo indicador está relacionado a enfoques de protección social en los cuales los mecanismos o sistemas públicos tienen un rol preponderante. Como se ve, aun utilizando la misma variable, los indicadores pueden ser muy diferentes y abrir nuestro análisis a unos temas pero obviando o ignorando otros. 
Una situación similar ocurre con la selección de técnicas para recolectar y analizar datos, incluyendo las fuentes de información. Por ejemplo, algunos estudios (implementados principalmente por agencias de cooperación financiera) que buscan calificar los niveles de "buena gobernanza" en un país determinado, utilizan como una de sus fuentes de información la 'percepción' de algunos profesionales expertos en relación a temas complejos como aumento o disminución en los niveles de corrupción, certeza jurídica, libertad, etc., (21). Es claro que la visión de unos cuantos "expertos" sobre atributos sociales muy complejos es de poca representatividad si se desea saber la percepción de la ciudadanía en relación a esas variables. En otros casos se utilizan datos provenientes de encuestas de opinión como el 'Latino barómetro" (22). Aun cuando los datos de esas encuestas tienen una mayor representatividad que la consulta de una selección de "expertos", todavía es limitada para poder recoger información a profundidad de algunos grupos poblacionales que usualmente no son cubiertos por las encuestas (población en extrema pobreza, institucionalizados y otros).

Existen pocos estudios que han hecho el esfuerzo por recolectar información acerca de la percepción sobre variables de gobernanza en población rural, población en extrema pobreza, desempleados, etc. Si los principios y valores que rigen la toma de decisión en los sistemas de salud son la equidad, solidaridad y la participación social, entonces también se debe recoger información de los grupos sociales que sufren directamente las consecuencias de las inequidad y que experimentan toda una serie de barreras para participar en forma efectiva en las políticas públicas.

Lo discutido en esta sección busca llamar la atención hacia el riesgo que existe cuando se hace uso de variables e indicadores que están disponible en la literatura sin antes indagar sobre el marco conceptual y normativo que fundamenta dichas variables e indicadores. Ello porque existe la posibilidad de incompatibilidad entre los principios y valores que se sostienen (por ejemplo equidad y solidaridad en el sistema de salud) y los principios y valores que sostienen algunos indicadores y variables de uso frecuente en los ejercicios de investigación. Por lo tanto, es necesario ser inquisitivo antes de aplicar en forma ciega los diferentes indicadores, variables y técnicas de recolección de datos.

\section{CONCLUSIONES}

La investigación alrededor de la gobernanza en sistemas de salud aborda aspectos $\mathrm{y}$ actores sociales novedosos que no son recogidos por los ejercicios tradicionales 
de investigación en sistemas y servicios de salud. Dicha investigación requiere un abordaje de variables que no se encuentran únicamente dentro del sector salud sino que principalmente en el contexto sociopolítico.

Si aceptamos la gobernanza como procesos de toma de decisión en el cual confluyen las relaciones de poder, entonces situaciones de consenso y conflicto entre los actores sociales y las organizaciones son inherentes a dichos procesos. Por lo tanto, un tema central en la investigación de la gobernanza en los sistemas de salud es la indagación sobre las reglas formales y no formales que rigen los procesos de toma de decisión y la modalidad en que se expresan los conflictos y llegan a resolverse (sea por consenso u otros mecanismos). También es importante indagar los recursos de poder que poseen los actores sociales que participan en procesos de toma de decisión y la capacidad de influencia (como expresión del poder) que logran generar.

Los procesos de toma de decisión en el sistema de salud, al igual que en el resto de políticas públicas, no suceden en el vacío sino dentro de un marco de principios éticos, motivaciones e incentivos que pueden ser explícitos o implícitos. Identificar y profundizar la comprensión de dichos aspectos es esencial. Al mismo tiempo, la investigación alrededor de la gobernanza no es 'neutral' sino que de manera explicita o implícita, se apoya en una serie de principios y valores que sustentan el cuerpo metodológico (variables, indicadores y técnicas de recolección y análisis de datos) que aplican los investigadores. Aun cuando los ejercicios de investigación en el tema de gobernanza pueden tener énfasis en el aspecto normativo o en el aspecto analítico, ambos son necesarios para un abordaje que busque indagar y comprender la complejidad de los fenómenos sociales (en este caso los procesos de toma de decisión alrededor de políticas públicas) y las formas en que se puede alcanzar las metas sociales que han definido los sistemas de salud (equidad en el acceso y financiamiento, ciudadanía en salud plena y eficiencia en el uso y asignación de los recursos públicos) •

Agradecimientos: $\mathrm{El}$ autor agradece los comentarios al manuscrito realizados por Lise Lamothe y Francoise Barten. Las ideas discutidas en el articulo fueron beneficiadas por las discusiones con otros colegas, particularmente Armando de Negri, Nancy Molina y el resto de colegas investigadores que participaron en dos talleres (Lima, Perú y Montreal, Canadá) organizados por el Governance, Equity and Health (GEH) del International Development Research Centre (IDRC). 


\section{REFERENCIAS}

1. Hewitt C [Internet]. Usos y Abusos del Concepto de Gobernabilidad. Revista Internacional de Ciencias Sociales. 1998; 155 (marzo). Disponible en http://www.unesco.org/issj/rics155/ alcantaraspa.html Consultado 12 de febrero 2007.

2. Hyden G, Court J, Mease K [Internet]. Making Sense of Governance: the Need for Involving Local Stakeholders. 2003. Disponible en http://www.odi.org.uk/resources/download/3135.pdf

3. Barten F, Pérez R, Espinoza E, Morales C. Democratic governance-fairytale or real perspective? Lessons from Central America. Environment \& Urbanization. 2002; 14(1):129-144.

4. Mukhopadhyay M. (Ed). Governing for Equity. Gender, Citizenship and Governance. Royal Tropical Institute. Amsterdam. KIT; 2003.

5. Prats J. El Concepto y el Análisis de la Gobernabilidad. Revista Instituciones y Desarrollo. 2003; 14-15:239-269.

6. PNUD [Internet]. Reconceptualizing Governance. Discussion Paper 2. Capitulo 1: Concepts of Governance and Sustainable Human Development. Management Development and Governance Division, Bureau for Policy and Programme Support. United Nations Development Programme. New York 1997. Disponible en: http://mirror.undp.org/magnet/ Docs/!UN98-21.PDF/!RECONCE.PTU/!sec1.pdf ConsultadoNoviembre del 2009.

7. Prats J [Internet]. Gobernabilidad Democrática para el Desarrollo Humano: Marco Conceptual y Analítico. Instituciones y Desarrollo. 2001 (9 octubre). Disponible en http://www.iigov.org/ id/article.drt?edi=187384\&art=187477 Consultado 2 de febrero del 2007.

8. WHO 1998 Good governance for health. WHO/CHS/HSS98.2. World Health Organization. Geneva.

9. Haugaard M. Power: a Reader. Manchester University Press; 2002.

10. Bourdieu P. Social Space and Symbolic Power. Sociological Theory 1989; 7 (1):14-25.

11. Giddens A. Sociología. 4ta edición. Madrid: Alianza Editorial; 2001.

12. Poulantzas N. Poder Político y Clases Sociales en el Estado Capitalista. México: Siglo XXI; 1968.

13. Arendt H. On violence. New York: Harcourt, Brace \& World; 1970.

14. Morris P. Power a Philosophical Analysis. 2da edición. Manchester: Manchester University Press; 2002.

15. Benclowicz JD [Internet]. La izquierda y la emergencia del movimiento piquetero en la Argentina. Análisis de un caso testigo. 2006. Disponible en http://www.publicaciones.cucsh.udg.m/ pperiod/espiral/espiralpdf/Espiral37/123-143.pdf Consultado Noviembre del 2008.

16. Carrillo R [Internet]. Movimientos sociales y hegemonía (2003). Disponible en http:// www.uasb.edu.ec/padh/centro/pdfs6/Ricardo\%20Carrillo.pdf Consultado: noviembre 2008.

17. Panchano S [Internet]. Ecuador: cuando la inestabilidad se vuelve estable (2005). Disponible en http://www.redalyc.uaemex.mx/redalyc/pdf/509/50902305.pdf Consultado Noviembre del 2008.

18. Court J, Hyden G, Mease K. Assessing governance: methodological challenges. World Governance Survey discussion paper 2. United Nations University; 2002.

19. Hufty M, Báscolo E, Bazzani R. Gobernanza en Salud: Un Aporte Conceptual y Analítico para la Investigación. Cad. Saúde Publica 2006; 22(sup): 34-45.

20. Prats J. La Cooperación Descentralizada a la Gobernabilidad y el Fortalecimiento Institucional Local. Institut Internacional de Governabilitat de Catalunya, Barcelona. España; 2004.

21. Kaufmann D, Kraay A. Governance Indicators: Where are We, Where should We be Going? Policy Research Working Paper 4370. The World Bank; 2007.

22. Corporación Latinobarómetro. Informe 2008. Santiago de Chile; 2008. 